\title{
Escoabilidade de leitos de partículas inertes com polpa de frutas tropicais. Efeitos na secagem em leito de jorro ${ }^{1}$
}

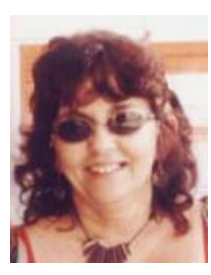

Maria de F. D. de Medeiros ${ }^{2}$, Odelsia L. S. de Alsina ${ }^{3}$, Sandra C. Rocha ${ }^{4}$, Carlos E. de M. Jerônimo ${ }^{5}$ Ana L. de M. L. da Mata², Uliana K. L. de Medeiros ${ }^{5}$ \& Andréa A. Furtunato ${ }^{5}$

1 Parte da tese de Doutorado do primeiro autor na FEQ/UNICAMP

2 UFRN. Av. Salgado Filho, s/n. Lagoa Nova, CEP 59072-970, Natal, RN. Fone: (84) 215-3756. E-mail: luiza@ufrnet.br, damata@brhs.com.br (Foto)

${ }^{3}$ DEQ/CCT/UFPB, Campus II, CEP 581092-970, Campina Grande, PB. Fone (83) 310-1115. E-mail: odelsia@deq.ufpb.br

${ }^{4}$ Departamento de Termofluidodinâmica, FEQ/UNICAMP, CEP13081-970, Campinas, SP. Fone: (19) 3788-3929. E-mail: rocha@feq.unicamp.br

${ }^{5}$ Curso de Engenharia Química da UFRN, Natal, RN. E-mail: carlos@eq.ufrn.br, anailu@hotmail.com, dea@eq.ufrn.br

Protocolo 037 - 29/3/2001

Resumo: Neste trabalho, foram caracterizados seis tipos de material inerte, utilizados na secagem de polpa de frutas em leito de jorro. Determinou-se o ângulo de repouso das partículas, com e sem adição de água e de polpa de diversas frutas tropicais. Correlacionou-se a escoabilidade com as propriedades das partículas e com a composição química das polpas. Analisou-se a influência do ângulo de repouso sobre o desempenho do secador, no que se refere à produção. Os resultados mostraram que, em geral, as polpas com elevadas concentrações de gordura e sólidos insolúveis e baixos teores de açúcares redutores, facilitam a escoabilidade. Uma análise dos resultados obtidos na secagem de polpa de frutas tropicais, utilizando-se partículas de poliestireno de baixa densidade, como material inerte, mostrou que, embora a escoabilidade permita a obtenção de menores vazões de jorro mínimo, em relação ao desempenho do secador, pode não favorecer uma produção maior de pó.

Palavras-chave: escoabilidade, ângulo de repouso, leito de jorro, polpa de frutas

\section{Flowability of inert particle beds with fruit pulp. Effects on the drying in spouted bed}

\begin{abstract}
In this work six types of inert particles were characterized and analyzed for drying tropical fruit pulps. The repose angle was determined with and without the addition of water and pulp of various tropical fruits. The bed flowability was related to the particle properties and chemical composition of pulps. The influence of the repose angle on the drying performance was analyzed. It was also verified that the composition of pulps influenced the bed flowability. The global analysis showed that the pulps with high lipids and insoluble solids content and low reducing sugar content improved the bed flowability. The results obtained with the drying of the fruit pulps using low-density polystyrene granules as inert particles showed that high flowabilities lead to lower minimum spout flow rates, but do not necessarily lead to the highest powder production.
\end{abstract}

Key words: flowability, repose angle, spouted bed, fruit pulps

\section{INTRODUÇÃO}

A secagem de pastas e suspensões utilizando-se secadores de leito de jorro com partículas inertes, tem sido bastante estudada nos últimos vinte anos. Este secador tem-se mostrado boa alternativa ao secador spray, por fornecer produtos de qualidade similar, a custos significativamente inferiores.

Os primeiros estudos sobre secagem de pastas e suspensões (cristalização evaporativa) num leito de jorro com partículas inertes, foram desenvolvidos por um grupo de pesquisadores do Instituto de Tecnologia de Leningrado, na secagem de lacas e tintas. A técnica vem sendo utilizada na secagem de diversos tipos de materiais, tais como sangue animal (Pham, 1983), leveduras, polpa de frutas (Alsina et al., 1996), leite, extratos farmacológicos, soluções de sal e açúcar, além de vários produtos químicos, com resultados bastante promissores (Passos et al., 1997).

Os primeiros trabalhos sobre secagem de polpa de frutas foram apresentados por Hufenussler \& Kachan (1985). Os autores estudaram a desidratação de purê de banana, analisando 
a qualidade do pó em relação ao obtido em secadores spray e a vácuo; promoveram, também, uma avaliação sensorial, na qual o sabor do purê reconstituído foi comparado com o purê original e com o produzido em secadores de tambores rotativos. Os resultados mostraram que o pó obtido no secador de leito de jorro atendia aos padrões de conservação exigidos e que o sabor do purê reconstituído não diferia do original a um nível de $5 \%$ de significância, nível este não atingido pelo purê reconstituído com o pó de banana obtido no secador rotativo.

No secador de leito de jorro, a atomização da pasta ou suspensão sobre o leito reveste as partículas com uma fina camada de material. A medida em que seca, a película se torna frágil, fragmentando-se devido aos efeitos de colisões interpartículas. O material na forma de pó é então arrastado para fora pela corrente de ar. Um ciclone conectado ao secador promove a separação e recolhimento do pó; entretanto, muitas vezes as taxas de remoção da película são muito baixas, promovendo acumulação do material no leito, sendo este um dos graves problemas deste secador, chegando até mesmo a inviabilizar sua utilização na secagem de diversos materiais. Estudos sobre a estabilidade de leitos de jorro em presença de líquidos foram realizados por Schneider \& Bridgwater (1993). Oliveira \& Passos (1997) simularam a secagem de suspensões em leito de jorro cônico.

Diversos trabalhos relacionados com a secagem de polpa de frutas em leito de jorro, vêm sendo apresentados nos últimos dez anos; Lima (1992) estudou a secagem da polpa de umbu, encontrando resultados bastante satisfatórios. Os estudos foram ampliados para a secagem em leito de jorro de polpa de cajá, manga, pinha, cajá-manga, serigüela e acerola (Ramos et al., 1997; Lima et al., 2000) dentre outros. Em todos esses trabalhos, os autores relatam problemas de acúmulo de material, com comprometimento das condições fluidodinâmicas e estabilidade do leito.

Martinez et al. (1995) apresentaram uma discussão detalhada sobre o comportamento do leito de jorro na secagem de produtos vegetais, relacionando os problemas de aderência das partículas e interrupção do jorro com as características aderentes do suco de frutas e vegetais, provocadas pelos elevados conteúdos de açúcar. Na conclusão do trabalho enfatizaram a necessidade de se aprofundar os estudos sobre os processos físico-químicos que ocorrem durante o processamento no secador de leito de jorro, com a finalidade de esclarecer as razões pelas quais ocorre a adesão do produto sobre as partículas inertes. Passos \& Mujumdar (2000) analisaram os efeitos das forças cohesivas em leito de jorro e fluidizado.

Lima et al. (2000) correlacionaram a composição de uma série de frutas tropicais com a eficiência de produção do pó, cujos resultados mostraram que havia influências isoladas dos teores de gordura, fibras e açúcares redutores. Observaram, ainda, o efeito combinado desses constituintes, no desempenho da secagem em leito de jorro.

Além da composição, outros fatores, como características da superfície e propriedades das partículas inertes (massa específica, esfericidade, ângulo de repouso) também interferem diretamente na remoção da película e, conseqüentemente, na acumulação de material no leito.

O ângulo de repouso é o ângulo de talude formado com a horizontal no qual o material permanecerá quando empilhado.
O tamanho, a forma, o teor de umidade e a orientação das partículas, exercem influência no ângulo de repouso. Diferenciam-se dois tipos de ângulo de repouso: o estático e o dinâmico. A designação estática refere-se à fricção do sólido granular sobre si mesmo, quando todo o material está estático. Nos casos em que o volume do material está em movimento, como nos descarregamentos, encontra-se envolvido o ângulo de repouso dinâmico. A fluidez de um material e o ângulo de repouso estão correlacionados, exercendo influência decisiva no projeto e na utilização dos silos de armazenamento. Considerando-se que materiais com baixa taxa de escorregamento não são propícios à secagem em secadores contínuos, a determinação do ângulo de repouso é muito importante para o projeto de secadores, já que interfere diretamente na sua configuração.

Este trabalho fornece dados importantes para a escolha do material inerte a ser utilizado na secagem de alimentos na forma de pasta ou suspensões em leito de jorro ou, ainda, em outros tipos de equipamentos que operem com leito de partículas inertes. O presente trabalho tem como objetivo analisar as interferências das concentrações de sólidos insolúveis (representados, na grande maioria, pelas fibras presentes nas polpas), de gorduras, de sólidos totais e de açúcares redutores, no ângulo de repouso dos leitos pesquisados. O efeito dessa variável sobre o comportamento do leito de jorro na secagem de polpa de frutas tropicais, é analisado através de dados de eficiência na produção de pó obtidos por Ramos et al. (1997) e vazão de jorro mínimo (Jerônimo et al., 2001 ) com o objetivo de correlacionar a escoabilidade do leito com o seu desempenho na secagem de polpa de acerola, cajá, umbu, seriguela, manga, pinha e abacate.

\section{MATERIAL E MÉTODOS}

Utilizou-se partículas de poliestireno de alta e baixa densidade (PSAD e PSBD), polietileno de alta e baixa densidade (PEAD e PEBD) e o SANGEL (copolímero de estireno e acetonitrila). Os materiais foram adquiridos junto às indústrias de polímeros (BASF, POLITENO e PROQUIGEL) devidamente caracterizados. Os resultados da caracterização do diâmetro médio das partículas $\left(\mathrm{d}_{\mathrm{p}}\right)$, da esfericidade $(\phi)$ e da massa específica $\left(\rho_{\mathrm{s}}\right)$, determinados, pelos métodos do volume deslocado, da área projetada e por picnometria, respectivamente, são mostrados na Tabela 1 .

Tabela 1. Característica dos materiais inertes utilizados

\begin{tabular}{cccccc}
\hline & PEBD & PEAD & PSBD & PSAD & SANGEL \\
\hline $\mathrm{d}_{\mathrm{p}}(\mathrm{m}) \times 10^{2}$ & 0,388 & 0,390 & 0,335 & 0,284 & 0,366 \\
$\phi$ & 0,75 & 0,76 & 0,59 & 0,52 & 0,64 \\
$\rho \mathrm{\rho s}\left(\mathrm{kg} \mathrm{m}^{-3}\right)$ & 917 & 950 & 1028 & 1030 & 1066 \\
\hline
\end{tabular}

PEBD: polietileno de baixa densidade

PEAD: polietileno de alta densidade

PSBD: poliestireno de baixa densidade

PSAD: poliestireno de alta densidade

As polpas foram obtidas por meio da trituração das frutas em processador doméstico, seguida de prensagem em peneiras domésticas de malha fina. Utilizou-se polpa de acerola, abacate, cajá, manga, serigüela, umbu e pinha.

De acordo com as Normas Técnicas e Analíticas do Instituto Adolfo Lutz (IAL, 1982) determinou-se o conteúdo de gordura, 
fibras, acidez, sólidos solúveis, umidade e o $\mathrm{pH}$ das polpas. $\mathrm{Na}$ determinação dos açúcares redutores e não redutores, foi utilizado o método de Somogyi (1952).

$\mathrm{O}$ ângulo de repouso foi determinado pela inclinação de uma plataforma horizontal. Amostras significativas do material eram espalhadas em camadas sobrepostas sobre a plataforma horizontal. Manteve-se uma camada completa da mesma espessura $(3 \mathrm{~cm})$ para todos os materiais; em seguida, variava-se lentamente o ângulo de inclinação da superfície, até se observar o início do deslocamento do material. A inclinação da superfície no momento em que o material começava a se mover, medida na escala acoplada à estrutura, foi designada como o ângulo de repouso. Um esquema do equipamento está representado na Figura 1. Foram efetuadas medidas dos ângulos de repouso para os materiais inertes sem e com a adição de polpas e de água. Na adição de polpas e água, utilizou-se uma percentagem fixa de $2 \%$ sobre o leito de inerte.

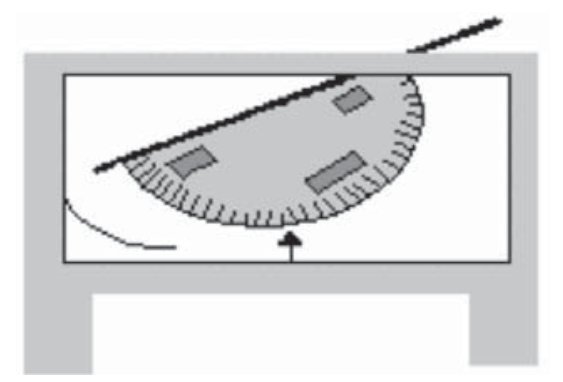

Figura 1. Módulo para medição do ângulo de repouso

Para se avaliar os efeitos da escoabilidade sobre o desempenho, os valores de ângulo de repouso obtidos neste trabalho foram correlacionados com dados de eficiência de produção de pó (Ramos et al. , 1997) e vazão de jorro mínimo (Jerônimo et al., 2001) na secagem de polpas de cajá, manga, umbu, pinha, seriguela e de cajá-manga em leito de jorro com partículas de poliestireno de baixa densidade, como material inerte. As características das partículas encontram-se na Tabela 1. As polpas das diversas frutas foram processadas em leitos de jorro convencional, com coluna de base cônica de $60^{\circ} \mathrm{com} 18 \mathrm{~cm}$ de diâmetro, $1 \mathrm{~m}$ de altura e relação diâmetro de coluna/diâmetro do orifício de admissão igual a 6. Os ensaios de secagem foram realizados em batelada e as curvas características fluidodinâmicas obtidas seguindo-se a metodologia usual. Maiores detalhes sobre a metodologia podem ser encontrados em Ramos et al. (1997) e Jerônimo et al. (2001).

\section{RESULTADOS E DISCUSSÃO}

Na Figura 2 são representados os valores obtidos para os ângulos de repouso dos leitos sem e com adição de água e de polpa. Conforme se observa, tanto a presença da água como a das polpas provoca aumento no ângulo de repouso, diminuindo, conseqüentemente, a escoabilidade das partículas. Com a polpa de acerola, todos os inertes estudados apresentaram maior ângulo de repouso. Com relação aos inertes, os menores ângulos de repouso foram obtidos com o polietileno, tanto de alta como de baixa densidade Esses materiais, quando caracterizados, apresentaram maiores esfericidades.

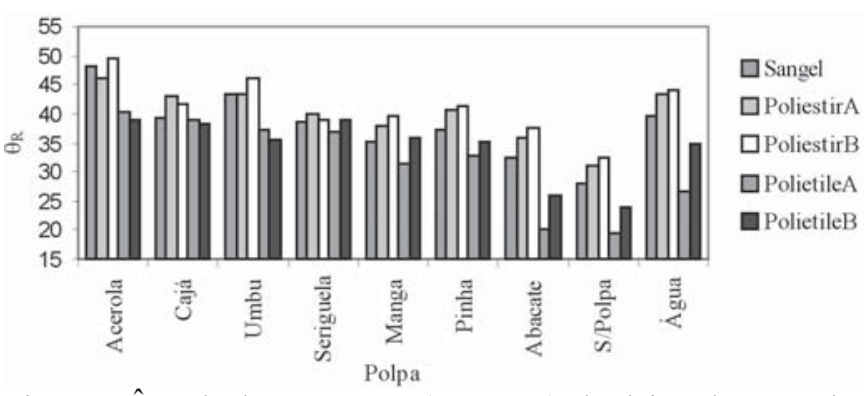

Figura 2. Ângulo de repouso, $\theta_{\mathrm{R}}$ (em graus), dos leitos de partículas inertes, com e sem adição de água ou polpa

No que diz respeito à influência das características dos materiais inertes sobre a escoabilidade, não foram encontradas tendências bem definidas com respeito a possíveis interferências da densidade do sólido ou diâmetro das partículas. A tendência observada em relação à esfericidade é mostrada na Figura 3 , onde se confirma, conforme era esperado, que o ângulo de repouso diminui com a esfericidade. A mesma figura ilustra a influência das polpas e da água na escoabilidade, em relação ao leito formado apenas com as partículas inertes.

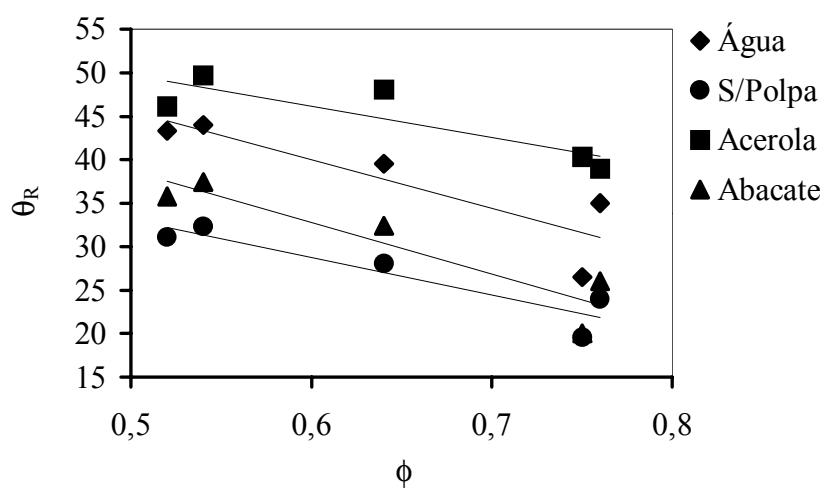

Figura 3. Efeito da esfericidade ( $\phi$, adimensional) e do tipo de material atomizado sobre o leito das partículas no ângulo de repouso $\left(\theta_{R}\right.$, em graus)

Embora se mantenha o decrescimento do ângulo de repouso com a esfericidade, as interferências das polpas e da água são diferentes. Com a polpa de acerola, os valores dos ângulos de repouso são sensivelmente mais elevados, demonstrando que a presença desta polpa dificulta, de forma bastante significativa, a escoabilidade das partículas. Além disso, a água também provoca aumento no ângulo de repouso, porém de forma mais atenuada que a acerola. Com relação ao abacate, o ângulo de repouso é pouco afetado pela presença desta polpa. Com as partículas mais esféricas, os ângulos de repouso dos leitos com adição da polpa de abacate, apresentaram valores muito próximos e até inferiores aos medidos com esses inertes (polietileno de alta e baixa densidade) sem adição de qualquer outro material.

Esses resultados sugerem forte interferência de outros constituintes das polpas, além da água, sobre a escoabilidade de leitos de partículas acrescidos desses materiais.

De acordo com Lima et al. (2000) as concentrações de fibras, açúcares redutores e gorduras, interferem na eficiência de produção de pó no processo de secagem de polpa de frutas em leito de jorro com partículas inertes. 
As Figuras 4 e 5 ilustram as influências das concentrações de sólidos insolúveis $\left(\mathrm{X}_{\mathrm{si}}\right)$, de gorduras $\left(\mathrm{X}_{\mathrm{g}}\right)$ e açúcares redutores $\left(\mathrm{X}_{\mathrm{AR}}\right)$ estas últimas expressas pelo fator $\mathrm{X}_{\mathrm{g}} / \mathrm{X}_{\mathrm{AR}}$, sobre o ângulo de repouso. Com a finalidade de se avaliar o efeito dessas concentrações para os diversos tipos de inerte, as relações de composição foram multiplicadas pela esfericidade das diferentes partículas $\left(\mathrm{X}_{\mathrm{si}} \phi, \mathrm{X}_{\mathrm{g}} \phi / \mathrm{X}_{\mathrm{AR}}\right)$.

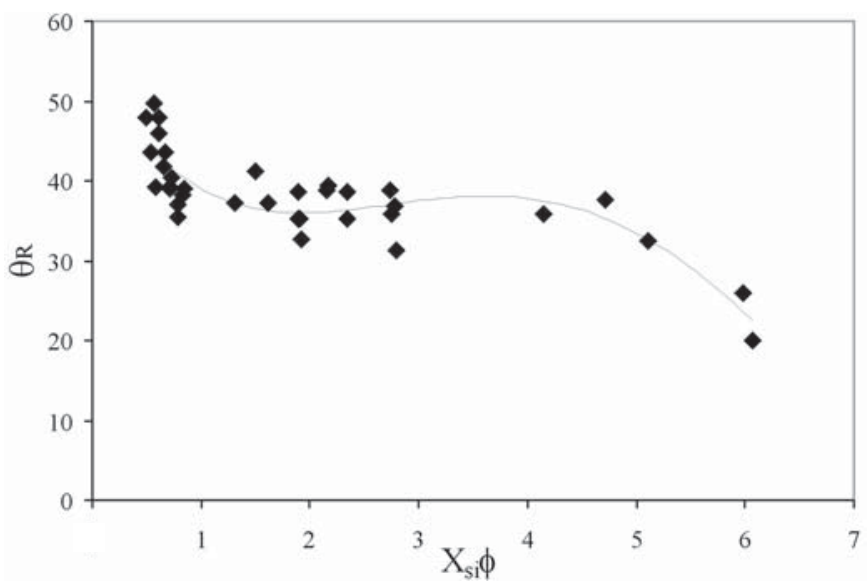

Figura 4. Influência do fator $\left(X_{\mathrm{si}} \phi\right)$ no ângulo de repouso $\left(\theta_{\mathrm{R}}\right.$, em graus)

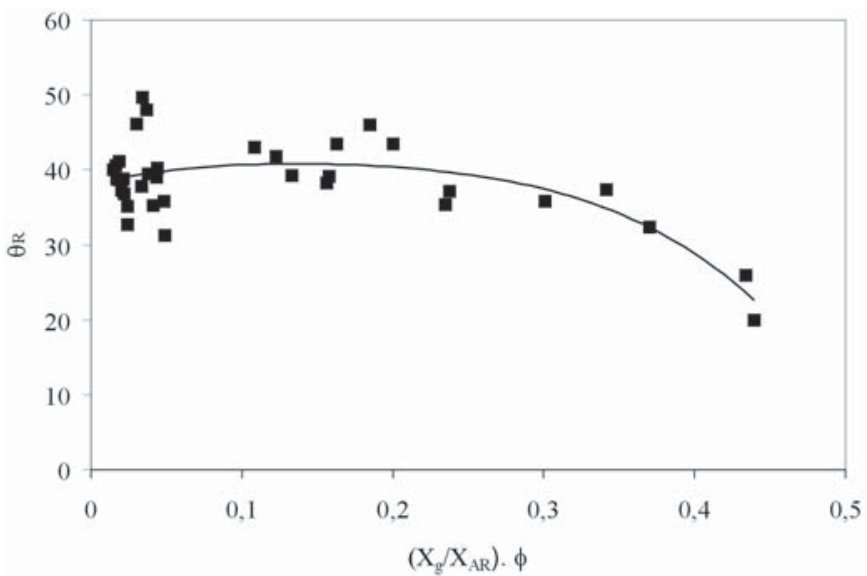

Figura 5. Influência do Fator $\left(\mathrm{X}_{\mathrm{g}} / \mathrm{X}_{\mathrm{AR}}\right) \phi$ no ângulo de repouso $\left(\theta_{\mathrm{R}}\right)$

Conforme se observa na Figura 4, que representa dados para todas as polpas e inertes, há tendência bem definida de aumento da escoabilidade ou diminuição do ângulo de repouso com a concentração de sólidos insolúveis devido, provavelmente, ao fato de que, com proporções fixas de polpa para inerte, uma maior concentração de sólidos insolúveis corresponde a uma presença menor de fase líquida; no entanto, esta afirmação entra em contradição com a observação de que com água pura a escoabilidade é maior que as observadas com o acréscimo de algumas polpas, o que leva a confirmar, mais uma vez, a interferência de outros fatores.

Uma hipótese a ser analisada é a de que os sólidos insolúveis podem agir como uma espécie de lubrificante, no caso das gorduras ou "rolamentos", no escoamento das partículas de inerte umas sobre as outras facilitando, assim, a escoabilidade do leito. De forma individual, foi observado polpas de frutas com elevadas concentrações de gordura, tal como a polpa de abacate, aumenta a escoabilidade das partículas. Paralelamente, polpas com elevados teores de açúcares provocam efeito inverso sobre a escoabilidade. A Figura 5 ilustra o comportamento do ângulo de repouso frente ao fator $\mathrm{X}_{\mathrm{g}} / \mathrm{X}_{\mathrm{AR}} \phi$, considerando-se todas as polpas e os materiais inertes estudados. Observa-se que em níveis superiores a $0,2 \%$, há diminuição do ângulo de repouso com a relação $\mathrm{X}_{\mathrm{g}} / \mathrm{X}_{\mathrm{AR}} \phi$, o que demonstra que, a partir de certo limite de concentração de gorduras, o efeito positivo das gorduras sobre a escoabilidade é predominante.

Ao se correlacionar os valores dos ângulos de repouso dos leitos acrescidos de polpa de frutas com os dados das variáveis fluidodinâmicas obtidos por Jerônimo et al. (2001) observa-se que a vazão de jorro mínimo $\left(Q_{j m}\right)$ cresce com o ângulo de repouso $\left(\theta_{\mathrm{R}}\right)$. Este comportamento se encontra ilustrado na Figura 6, com dados de Jerônimo et al. (2001) que trabalharam com leitos de poliestireno de baixa densidade e com as mesmas polpas, cujos efeitos sobre a escoabilidade são estudados neste trabalho.

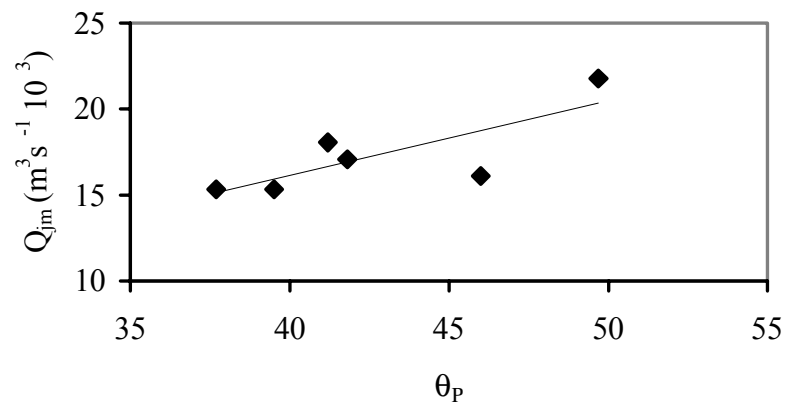

Figura 6. Influência do ângulo de repouso $\left(\theta_{R}\right)$ na vazão de jorro mínimo $\left(Q_{j m}\right)$. Figura construída a partir de dados de vazão de jorro mínimo de Jerônimo et al. (2001)

Na Figura 7 encontra-se ilustrado o efeito do ângulo de repouso sobre a eficiência de produção de pó $\left(\mathrm{E}_{\mathrm{f}}\right)$. Os dados relativos à eficiência foram obtidos por Ramos et al. (1997) na secagem de polpa de frutas tropicais, utilizando partículas de poliestireno de baixa densidade, como material inerte.

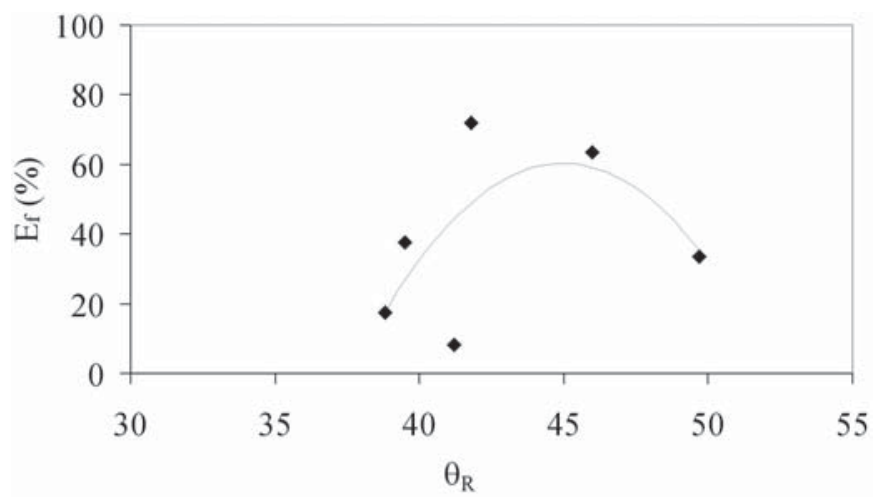

Figura 7. Influência do ângulo de repouso $\left(\theta_{R}\right)$ na eficiência de produção de pó (Ef) da secagem, de polpa de frutas com leito inerte de poliestireno de baixa densidade. Dados de eficiência extraídos de Ramos et al. (1997)

Conforme se observa na Figura 7, o comportamento é complexo, com tendência a passar por um ponto que corresponde a um máximo de eficiência. Este máximo pode ser 
justificado pelo atrito necessário para romper a película de material aderido à superfície das partículas.

Quando a escoabilidade é muito elevada (baixos ângulos de repouso) há menos atrito entre as partículas, o que dificulta a liberação da película e, conseqüentemente, a produção de pó. Por outro lado, quando o ângulo de repouso é muito elevado, o movimento das partículas no leito fica prejudicado, havendo formação de zonas estagnadas, com comprometimento da fluidodinâmica do leito, problemas de instabilidade e diminuição da concentração de partículas na fonte. Esses problemas também afetam a produção de pó e podem chegar a provocar o colapso do jorro, inviabilizando o processo de secagem. Deve existir, portanto, dependendo do material que se deseja desidratar, um valor do ângulo de repouso que otimiza a eficiência de produção de pó. Considera-se, entretanto, a necessidade de se aprofundar os estudos sobre a relação entre o ângulo de repouso e os fatores relacionados com aderência da película, retenção de material no leito e absorção de água pelo material inerte, para melhor compreensão dos comportamentos observados e dos resultados preliminares obtidos neste trabalho.

Na Figura 8, correlacionam-se os dados de eficiência de produção de pó obtidos por Ramos et al. (1997), definida pela Eq. (1), com um novo fator expresso pela relação $\left[\mathrm{X}_{\mathrm{g}} /\left(\mathrm{X}_{\mathrm{AR}} \theta_{\mathrm{R}}\right)\right]$.

$$
E f=\frac{M_{\text {pó }}\left(1-U_{\text {pó }}\right) 100}{M_{\text {polpa }}\left(1-U_{\text {polpa }}\right)}
$$

donde:

Mpó - massa de pó coletada (g)

Upó - umidade do pó ( $\mathrm{g} \mathrm{g}^{-1}$, base úmida)

Mpolpa - massa de polpa alimentada ( $\mathrm{g}$ )

Upolpa - umidade da polpa ( $\mathrm{g} \mathrm{g}^{-1}$, base úmida)

Ajustou-se uma equação que relaciona a eficiência com o logaritmo deste fator. A Eq. (2) ajustada aos dados experimentais, apresentou coeficiente de correlação (r) da ordem de 0,93.

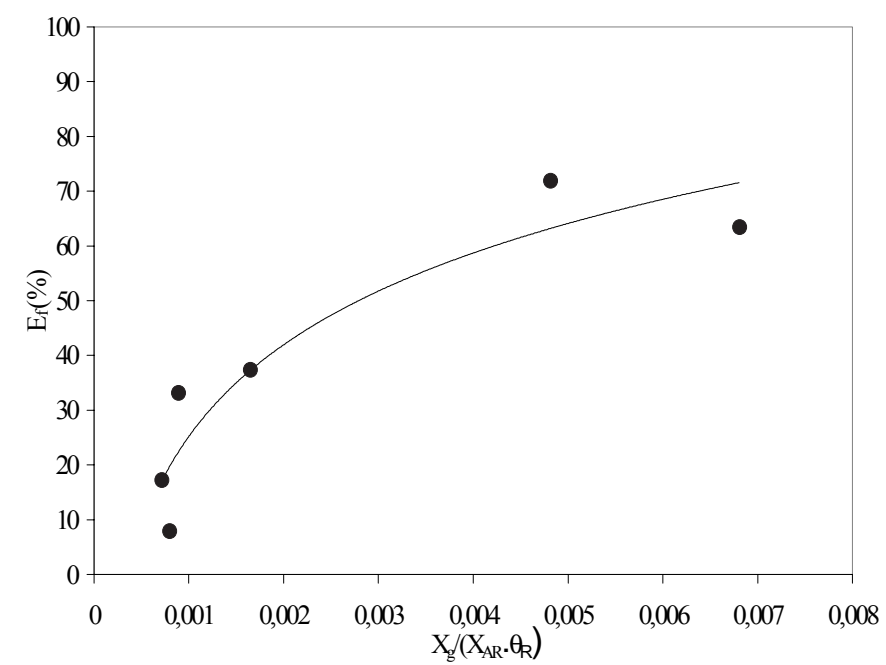

Figura 8. Influência do fator $\mathrm{X}_{\mathrm{g}} / \theta_{\mathrm{R}} \cdot \mathrm{X}_{\mathrm{AR}}$ na eficiência de produção de pó (Ef) na secagem de polpas de frutas com leito inerte de poliestireno de baixa densidade em leito de jorro. Obs.: Dados de eficiência extraídos de Ramos et al. (1997)

$$
E_{f}=24,176 \ln \left(\frac{X_{g}}{\theta_{R} X_{A R}}\right)+19,219
$$

\section{CONCLUSÕES}

Os resultados do presente trabalho conduzem as seguintes constatações e conclusões:

1. Dos materiais empregados como inertes na secagem de polpa de frutas em leito de jorro, o polietileno, principalmente o de alta densidade, apresentou a melhor escoabilidade ou menor ângulo de repouso, enquanto o poliestireno apresentou os maiores ângulos.

2. O ângulo de repouso de todos os leitos estudados, formados pelos diferentes polímeros com e sem adição de água ou polpa de frutas, diminui linearmente com a esfericidade das partículas. Para a faixa de tamanho das partículas e para os tipos de polímeros utilizados nos ensaios, não foram identificadas interferência da massa específica do sólido nem do diâmetro das partículas sobre o ângulo de repouso.

3. Tanto a água como as polpas de frutas interferem no ângulo de repouso. Para todos os polímeros estudados, os maiores ângulos de repouso foram encontrados depois da adição da polpa de acerola e a polpa de abacate foi a que promoveu a melhor escoabilidade.

4. Há uma nítida interferência da composição das frutas sobre o ângulo de repouso.

5. Menores ângulos de repouso promovem menores vazões de jorro mínimo.

6. Dados da eficiência de produção de pó foram correlacionados com um novo fator expresso pelo produto entre a razão de composição $\left[\mathrm{X}_{\mathrm{g}} /\left(\mathrm{X}_{\mathrm{AR}} \theta_{\mathrm{R}}\right)\right]$ e o ângulo de repouso. A eficiência de produção de pó apresentou-se como uma função logarítmica deste novo fator.

\section{LITERATURA CITADA}

Alsina, O.L.S.; Morais, V.L.M.; Lima, L.M.R.; Soares, F.H.L. Studies on the performance of the spouted bed dryer for the dehydration of West Indian cherry pulp. In: Drying'96. Mujumdar, A.S. ed.. Hemisphere Publishing Corp., New York, p.867-872. 1996

Hufenussler, M.; Kachan, G.C. Secagem de purê de banana em um secador de leito de jorro. In: Encontro sobre Escoamento em Meios Porosos, 12, 1985, São Paulo. Anais... São Paulo: USP, 1985. v.2, p.340-354.

IAL - Instituto Adolfo Lutz. Métodos químicos e físicos para análise de alimentos. v.1. 3.ed. São Paulo. São Paulo: Coordenadoria dos Serviços Técnicos Especializados- Secretaria do Estado da Saúde. 1985.244p.

Jerônimo, C.E.M.; Lima, M.F.M.; Da Mata, A.L.M.L; Alsina, O.L.S.; Rocha, S.C.S. Secagem de polpas de frutas em leito de jorro. Influência dos teores de gordura, açúcares e fibras nas variáveis fluidodinâmicas. In: Congresso Brasileiro de Engenharia Química Iniciação Científica.3, 1999, Belo Horizonte.Anais...Belo Horizonte: UFMG, 2001. CD Rom

Lima, M.F.M. Desidratação de polpa de umbu em leito de jorro - Estudos fluidodinâmicos e térmicos. Campina Grande: UFPB, 1992. 132p. Dissertação Mestrado 
Lima, M.F.M.; Rocha, S.C.S.; Alsina, O.L.S; Jerônimo, C.E.M; da Mata, A.L.M.L. Influência da composição química dos materiais no desempenho do processo de secagem de polpas de frutas em leito de jorro. In: Congresso Brasileiro de Engenharia Química, 14, 2000, Águas de São Pedro. Anais... Campinas: UNICAMP, 2000. CD Rom.

Martinez, O.L.A.; Brennam, J.G.; Nirajam, K. Estudio del secado de alimentos en um secador de fuente com partículas inertes. In: Congresso Ibero-americano de Alimentos, 1, 1995, Campinas. Anais... Campinas: UNICAMP, 1995. p.72-81.

Oliveira, J.M.; Passos, M.L. Simulation of drying suspentions in a conical spouted-bed. Drying Technology, Monticello NY, v.15, n.2, p.593-604. 1997.

Passos, M.L.; Massarani, G.; Freire, J.T.; Mujumdar, A.S. Drying of pastes in spouted beds of inert particles: Design criteria and modeling. Drying Technology, Monticello NY v.15, n.2, p.605-624. 1997.
Passos, M.L.; Mujumdar, A.S. Effect of cohesive forces on fluidized and spouted bed of wet particles. Powder Technology, Lausanne, v.110, p.222-238, 2000.

Pham, Q.T. Behavior of a conical spouted-bed dryer for animal blood. The Canadian Journal of Chemical Engineering, Oxford, v.61, n.3, p. 426-434. 1983

Ramos, C.M.P.; Lima, M.F.M.; Maria, Z.L. Polpa de frutas desidratadas em leito de jorro. Influência da composição química no desempenho do processo. Natal: UFRN, 1997. 75 p. Relatório Interno

Schneider, T.; Bridgwater, J. The stability of wet spouted beds. Drying Technology, Monticello NY, v.11, n.2, p.277-301. 1993.

Somogyi, M. Notes on sugar determination. Journal of Biological Chemistry, London, v.195, p.19-23. 1952. 